\title{
HIBRIDACIÓN ARTIFICIAL EN BERENJENA (Solanum melongena L.): EFECTO SOBRE LA PRODUCCIÓN DE FRUTOS Y SEMILLAS
}

\section{ARTIFICIAL HYBRIDIZATION OF EGGPLANT (Solanum melongena L.): EFFECT ON FRUIT AND SEED PRODUCTION}

\author{
Hermes Araméndiz Tatis ${ }^{1}$ \\ Carlos Cardona Ayala ${ }^{2}$ \\ Dairo Pérez Polo ${ }^{3}$
}

\section{RESUMEN}

La berenjena es una hortaliza con prospectiva de mercado nacional e internacional, por sus efectos positivos sobre la salud humana. En Colombia presenta limitaciones tecnológicas en materia de cultivares mejorados para las condiciones tropicales. El estudio se realizó en la Universidad de Córdoba, con el objetivo de identificar el momento óptimo del botón floral, para la hibridación artificial conducente a la formación de frutos y producción de semillas híbrida para uso por parte de los productores. Se utilizaron los cultivares Brinjal Súper Mohini (hipocótilo verde y fruto blanco) y Taiwán (hipocótilo y fruto púrpura), como parentales femenino y masculino, respectivamente. El diseño fue bloques completos aleatorizados con ocho tratamientos y cuatro repeticiones. Los tratamientos consistieron en combinar distintos estados de desarrollo de la flor; como parte femenina: botón floral de corola color crema (pre-antesis), botón floral de corola color morado claro (próximo a antesis) y botón floral de corola color morado

${ }^{1}$ Ph.D. Universidad de Córdoba, Grupo de Investigación en Cultivos Tropicales de Clima Cálido, carrera 6 No 76-103, Montería, Córdoba. e-mail: haramendiz@hotmail.com

2 M.Sc. Universidad de Córdoba, Grupo de Investigación en Cultivos Tropicales de Clima Cálido, carrera 6 № 76-103, Montería, Córdoba. e-mail: ccardona@sinu.unicordoba.edu.co

${ }^{3}$ Ingeniero Agrónomo. Universidad de Córdoba, Grupo de Investigación en Cultivos Tropicales de Clima Cálido, carrera 6 № 76-103, Montería, Córdoba. e-mail: dairoperez@yahoo.es (iniciando antesis) y como parte masculina: botón floral de corola color morado claro (próximo a la antesis), botón floral de corola color morado (iniciando antesis) y flor totalmente abierta, a las 8:00a.m. Con botones florales color crema (pre-antesis), no se obtuvo éxito en la hibridación artificial, en tanto que en las combinaciones de botones color morado claro (pre antesis), morado (iniciando antesis) y flor totalmente abierta fue efectiva la hibridación, sin diferencia significativa entre las combinaciones exitosas probadas; el mismo resultado estadístico, se obtuvo para peso de fruto, número de semillas por fruto e índice de semillas.

Palabras clave: Hortaliza, botón floral, antesis, peso de fruto, índice de semilla.

\section{SUMMARY}

The eggplant, because of its positive effects on human health, is a vegetable with prospective domestic and international markets. In Colombia the crop presents technological limitations, lacking improved cultivars for the tropical conditions. This study was conducted at the University of Cordoba, Montería, with the aim of identifying the optimal flower bud development for artificial hybridization, which should lead to the formation of fruit and seed production. Cultivars Brinjal Super Mohini (hypocotyl green and white fruit) and Taiwan (hypocotyl and purple fruit) were used as female and male parents, respectively. A complete randomized block design with eight treatments and four replications was employed. The treatments consisted in the combination of various 
stages of flower development; as female part: cream coloured flower bud corolla (pre-anthesis), clear purple coloured flower bud corolla (near anthesis) and purple coloured flower bud corolla (beginning anthesis); as male part: corolla of the flower bud slightly purple coloured (near anthesis), purple coloured (beginning anthesis) and the fully open flower at 8:00 am. With creamy flower buds (pre-anthesis) no success in artificial hybridization was obtained, while with the combinations of slightly purple buds (pre-anthesis), purple buds (beginning anthesis) and fully open flower the hybridization was effective, but without significant differences between the successful combinations tested. The same statistical result was obtained for fruit weight, number of seeds per fruit and seed index.

Key words: Vegetable, flower bud, anthesis, fruit weight, seed index

\section{INTRODUCCIÓN}

La berenjena (Solanum melongena L.) es la Solanácea hortícola de mayor importancia en el Caribe colombiano, después del ají y el tomate. Es originaria de las áreas tropicales del oriente, cultivada desde hace más de 4000 años por los chinos y árabes (Prohens et al. 2005) y su centro de diversidad genética es la India e Indochina (Sekara et al. 2007). Posee importancia económica y social en la cultura sinuana, ya que fue introducida por los árabes en el siglo pasado y, a partir de la variabilidad genética de las introducciones, fueron seleccionadas las plantas de mayor adaptación, contribuyendo al enriquecimiento de la base alimentaria de esta región del país, junto con sus costumbres, tradiciones y diversidad cultural (Aramendiz et al. 2006).

Es la principal hortaliza del caribe húmedo colombiano y es cultivada con variedades criollas que aún conservan características no deseables, como plantas altas, presencia de espinas en las hojas y cáliz, cojines florales hasta de cinco flores andromonóicas, de frutos de tamaño, color y forma variable, que inciden fuertemente en el rendimiento; se conocen como "criolla lila", "criolla negra", "criolla morada", "criolla blanca", las cuales, aparecen en cualquier cultivo, como resultado de las mutaciones, hibridación natural y selección realizada por el hombre con las introducciones realizadas por los árabes. Sus rendimientos oscilan entre 12 y $36 \mathrm{t} \cdot \mathrm{ha}^{-1} \mathrm{con}$ reducciones en sus rendimientos de 2,31, 6,05 y 9,11\%, en los últimos años, en los departamentos de Bolívar, Sucre y Córdoba, respectivamente (Agronet, 2008), en donde se siembran 260 ha que representan el $81 \%$ de las 319ha cultivadas en el país en 2005 (Agronet, 2008) y en áreas pequeñas que median entre $2.500 \mathrm{~m}^{2} \mathrm{y} 10.000 \mathrm{~m}^{2}$. Esta situación coloca en desventaja nuestros productores para competir con otros países, aún bajo el marco de tratados de libre comercio y/o acuerdos comerciales preferenciales, lo que amerita, buscar alternativas que contribuyan a superar esta barrera tecnológica.

La demanda de alimentos con mayor calidad está en aumento en el mundo, en particular en los países con más altos ingresos, como consecuencia de un mayor conocimiento de los consumidores de la relación entre una buena dieta y la salud (Acuerdo de competitividad de la cadena de hortalizas, 2006). En este sentido, la berenjena es una de las hortalizas con demanda creciente por parte de los consumidores de productos saludables y con propiedades medicinales, ya que posee propiedades reductoras del nivel de colesterol y de buen nivel nutricional (Filgueira, 2000; Bletsos, 2002), lo cual ha repercutido en las exportaciones que pasaron de 14.857t, en 1961 a 347.676t, en 2004, con un área de cultivo muy estática en los últimos años, destacando en América, a México y Honduras, como grandes exportadores (FAO, 2006; Agronet, 2008), donde el cambio varietal, por el uso de híbridos con rendimientos que oscilan entre $40 \mathrm{y} 50 \mathrm{t} \cdot \mathrm{ha}^{-1}$, con características de precocidad, tamaño y color de fruto uniforme, que apetecen al consumidor (Antonini et al. 2002; Sekara et al. 2007) y la producción ecológica, han contribuido a su posicionamiento en el mercado exterior, puesto que la población mundial quiere consumir alimentos sanos y nutritivos, convirtiendo la producción hortícola ecológica de berenjena en un sector fuerte, organizado y competitivo en el mercado mundial.

El conocimiento de la estructura floral y de la biología reproductiva en una especie es básico para el mejorador de plantas, con el fin de desarrollar las técnicas de emasculación e hibridación. De acuerdo con Gomes et al. (2001), el rápido progreso logrado en el mejoramiento genético de plantas, obedece al estudio de los proceso reproductivos que ocurren en las flores.

La berenjena es reportada en la literatura colombiana con porcentajes de polinización cruzada variables; es así como Jaramillo $\mathcal{E}$ Lobo (1990) señalan un 5\%, en 
tanto que Vallejo \& Estrada (2002), indican que este valor oscila entre 5 y 20\%. Aramendiz et al. (2006), obtuvieron valores entre 66 y $87 \%$ en el valle del Sinú, calificándola como prevalentemente alógama. En el continente asiático, los registros de otros investigadores fluctúan entre 10 y 29\% (Swarup, 1995).

La heterostilia es un polimorfismo floral controlado genéticamente, caracterizado por la presencia de dos o tres morfologías florales: distília y tristília, en las cuales se presenta longitud del estilo y filamento variables, al igual que el tamaño y producción de polen y de las papilas estigmáticas, típico de Solanácea y Rubiaceae (Kohn $\mathcal{E}$ Barrett, 1992; Motten E Stone, 2000; Texeira E Machado, 2004). Pérez (2006) reportó la presencia de distilia y tristília, en dos cultivares de berenjena en el valle del río Sinú, señalando que las flores brevistílicas acusaron esterilidad, a causa de papilas estigmáticas poco desarrolladas y bajo contenido de azúcar (Rylski et al. 1984).

Sun et al. (1990), Passam E Khah (1992) y Lee et al. (2002) señalan que además de la heterostília, existen factores ambientales que limitan la polinización, como la temperatura, la relación planta - agua y alta humedad relativa. Así mismo, la antesis es afectada por factores ambientales (Malm $\mathcal{E}$ Rachie, 1971) y el tiempo requerido para la apertura y el cierre de las flores es afectado por la temperatura y la humedad relativa (Siles et al. 2001). Sin embargo, en berenjena la heterosis viene siendo explotada económicamente por la facilidad de obtener semilla híbrida, por el tamaño grande de sus órganos florales, que facilita el proceso de emasculación y polinización controlada (Cardoso, 2001), período amplio de floración y gran cantidad de semillas por fruto (Aramendiz et al. 1999; Maluf, 2001).

La producción de semilla híbrida de Solanáceas involucra el mantenimiento de dos líneas parentales separadas (masculina y femenina). La masculina actúa como dador de polen y la femenina como receptor de polen. Las flores del progenitor femenino son emasculadas durante el estadio del botón floral óptimo para la polinización manual, cuyas anteras son removidas por medio de pinzas, que son polinizadas manualmente $\mathrm{y}$, consecuentemente, forman frutos que contienen las semillas híbridas (George, 1999).

El uso de híbridos $\mathrm{F}_{1}$ de Solanáceas viene ocurriendo desde hace muchos años en la agricultura de esta hortaliza, en razón a sus ventajas de mayor vigor de la planta, más resistencia a plagas y enfermedades, maduración precoz, homeostasis, mejor calidad y rendimiento de fruto (Antonini et al. 2002; Blat et al. 2007) y además mayor uniformidad de fruto. Estudios comparativos entre cultivares híbridos y variedades realizados por Sousa et al. (1997) y Antonini et al. (2002), donde evaluaron el rendimiento total y calidad de frutos y evidenciaron mayor rendimiento en los híbridos a causa de la heterosis y mayor cantidad de frutos de primera categoría.

Por tanto, el objetivo de este trabajo fue estudiar la hibridación artificial en berenjena, bajo las condiciones del Valle del Río Sinú, en función del peso y del número de semillas por fruto, para su aplicación en la obtención de híbridos $F_{1}$, que permitansu usopara el mejoramiento del rendimiento y la calidad de fruto.

\section{MATERIALES Y MÉTODOS}

El estudio, se realizó en el área experimental de la Universidad de Córdoba (Montería-Colombia), ubicada a una altura de $13 \mathrm{msnm}$. Sus coordenadas geográficas corresponden a los $9^{\circ} 26^{\prime} 16^{\prime \prime}$ y $7^{\circ} 22^{\prime}$ '05" de latitud norte y $74^{\circ} 47^{\prime} 43^{\prime \prime}$ y $76^{\circ} 30^{\prime} 01^{\prime \prime}$ de longitud oeste. La zona de vida a la cual corresponde la capital del departamento, Montería, se denomina bosque seco tropical (bs-T), según la clasificación de Holdrige, con precipitación anual de $1346 \mathrm{~mm}$, temperatura media del aire de $27,4^{\circ} \mathrm{C}$, humedad relativa de $84 \%$ y brillo solar anual de 2108,2 horas (Palencia et al. 2006).

Para las hibridaciones artificiales, se cruzaron los cultivares Brinjal Súper Mohini (hipocótilo verde y fruto blanco) y Taiwán (hipocótilo y fruto púrpura), de origen asiático y poseedores de marcadores genéticos contrastantes, tanto en el hipocótilo como en el color de fruto, como fuente femenina y masculina, cuyas corolas en los estados iniciales de desarrollo son de color blanco y cambian a color lila (morado), a medida que se aproximan a la antesis. Estos cultivares fueron introducidos a Colombia, por donación del gobierno de Taiwán y no son cultivados en nuestro medio.

Se utilizó el diseño de bloques completos aleatorizados con ocho tratamientos y cuatro repeticiones (Tabla 1). Cada unidad experimental estuvo constituida por cinco cruzamientos. 
Tabla 1. Estructura de tratamientos, según los estados de desarrollo del botón floral de la berenjena (Solanum melongena L.).

\begin{tabular}{|c|c|c|}
\hline TRATAMIENTO & PROGENITOR FEMENINOS & PROGENITOR MASCULINO \\
\hline 1 & Botón en preantesis* & Botón próximo a antesis ${ }^{\star \star}$ \\
\hline 2 & Botón en preantesis* & Botón iniciando antesis ${ }^{\star \star *}$ \\
\hline 3 & Botón en preantesis* & Flor totalmente abierta ${ }^{* \star * *}$ \\
\hline 4 & Botón próximo a antesis** & Botón próximo a antesis** \\
\hline 5 & Botón próximo a antesis ${ }^{* *}$ & Botón iniciando antesis ${ }^{* * *}$ \\
\hline 6 & Botón próximo a antesis ${ }^{* *}$ & Flor totalmente abierta*** \\
\hline 7 & Botón iniciando antesis ${ }^{* * *}$ & Botón próximo a antesis** \\
\hline 8 & Botón iniciando antesis ${ }^{* * *}$ & Botón iniciando antesis ${ }^{* * *}$ \\
\hline
\end{tabular}

* Botón con corola de color crema; ** botón con corola de color morado claro; ${ }^{* * *}$ botón con corola de color morado (a las 5:30 a 6:30 a.m.); ${ }^{* * * *}$ flor totalmente abierta (antesis completa a las 8:00a.m).

En cada cruzamiento, se realizó previamente la emasculación del progenitor femenino y una remoción de sépalos y pétalos; el polen del cultivar de hipocótilo púrpura, se colectó con un vibrador manual y con la ayuda de un pincel, se colocó sobre el estigma del cultivar de hipocótilo verde. Cada unidad experimental fue etiquetada inicialmente con el nombre de la madre seguido del padre, repetición, fecha y nombre de la persona quien hizo el cruzamiento. Terminada la polinización artificial, se protegió con un cartucho de papel y se hizo un seguimiento a cada una de las hibridaciones, por cinco días y se cosechó cada cruzamiento cuando el fruto alcanzó su grado de madurez indicado por el color de fruto amarillo; posteriormente, se sacaron las semillas y se eliminaron aquellas que flotaron en la superficie del agua por ser vanas o sin cavidad embrionaria (Brasil, 1992) y, luego, se sometieron a secamiento al aire bajo sombra por 36 horas en condiciones ambientales locales (Lanteri et al. 1990; Demir et al. 2005). De igual manera, la semilla $\mathrm{F}_{1}$ de cada cruzamiento fue sembrada en semillero, con el fin de verificar la efectividad a través del uso del marcador genético color del hipocótilo, donde el rojo es dominante sobre el verde, por medio del conteo de plántulas de hipocótilo color rojo (Kalloo, 1993).

Las siguientes variables de respuesta fueron evaluadas: peso promedio de frutos a madurez fisiológica (coloración dorada), número de semillas por fruto e índice de semillas (número de semillas en $1000 \mathrm{~g}$ ). Para todas las variables de estudio fue realizada transformación de datos log $(X+5)$. Posterior al análisis de varianza, las medias fueron comparadas por la prueba de Bonferroni; de igual manera, se estimaron correlaciones fenotípicas entre el peso de fruto, número de semillas por fruto e índice de semillas.

\section{RESULTADOS Y DISCUSIÓN}

Los tratamientos en los cuales se utilizaron como progenitores femeninos, los botones florales en estado de preantesis (1, 2 y 3), acusaron ausencia de éxito en la polinización artificial, posiblemente por falta de cambios morfológicos, menor turgencia de las papilas y falta de secreciones en las mismas, que no facilitan la germinación del polen y crecimiento del tubo polínico, para llegar a la nucela de los óvulos (Peñaloza, 2001), por lo que estos no fueron involucrados en el análisis de varianza.

La falla en el proceso de hibridación de los botones florales en preantesis (con corola de color crema) obedece a la falta de receptividad estigmática, verificada por la ausencia de efervescencia a la aplicación de peróxido de hidrógeno al 3\%, ya que en este estado de desarrollo, tanto en las papilas como en estilos y ovarios, no han ocurrido cambios bioquímicos y fisiológicos fundamen- 
tales para que los contenidos de proteína, azúcar, almidón y hormonas favorezcan la germinación de polen, como lo anotan Dulberger et al. (1981) y Gillaspy et al. (1993). Así mismo, este período ocurre al momento de la antesis por dos o tres días dependiendo del cultivar y de la especie (Bezerra \& Machado, 2003; Ofosu-Anim et al. 2006).

El análisis de varianza para las variables peso promedio de fruto a madurez fisiológica, número de semillas por fruto e índice de semillas (Tabla 2), acusó ausencia de diferencia significativa entre tratamientos $(P>0,05)$. Los valores altos de las varianzas de los errores experimentales condujeron a coeficientes de variación elevados, lo que no permitió detectar diferencias significativas entre tratamientos. Esta variación encontrada en este tipo de estudio ha sido reportada por otros investigadores y se ha explicado por la variación de las cantidades de polen depositadas sobre cada estigma y su incidencia en el crecimiento y en el desarrollo de frutos y semillas (Muniz et al. 2001; Polverente et al. 2005; Cardoso, 2005).

Tabla 2. Cuadrados medios del análisis de varianza para las variables peso de fruto (P.F.), número de semillas por fruto (N.S.P.F.) e Índice de semilla (I.S.), consideradas en la hibridación artificial en berenjena.

\begin{tabular}{|c|c|c|c|c|}
\hline \multirow{2}{*}{$\begin{array}{l}\text { Fuentes de } \\
\text { variación }\end{array}$} & \multirow{2}{*}{$\begin{array}{l}\text { Grados de } \\
\text { libertad }\end{array}$} & \multicolumn{3}{|c|}{ Cuadrados medios } \\
\hline & & P.F & N.S.P.F. & I.S \\
\hline Bloques & 3 & 4.455342 & 8.45525 & 47.15753 \\
\hline Tratamientos & 4 & 3.369842 n.s & 5.46594 n.s & $22.26324 n . s$ \\
\hline Error & 12 & 3.269916 & 6.17473 & 26.90775 \\
\hline Total & 19 & & & \\
\hline \multicolumn{2}{|c|}{ Media } & 121.94 & 620.75 & 139.03 \\
\hline C.V.(\%) & & 47 & 52 & 64 \\
\hline
\end{tabular}

C.V.= Coeficiente de variación; n.s. $=$ no significativo

Los valores medios de los tratamientos correspondientes a los estados de desarrollo del botón floral próximo a antesis (corola de color morado claro) y botón floral iniciando antesis (corola de color morado), para peso de fruto y número de semillas por fruto, sugieren un posible efecto del estado de desarrollo del botón floral sobre el peso del fruto y, por ende, la cantidad de semilla en los mismos, ya que las de más avanzado estado de desarrollo (iniciando antesis), registraron mayor peso promedio de frutos y número de semillas por fruto. Para ambas variables, los resultados son similares a los reportados por Polverente et al. (2005), quienes además señalan que la manipulación de las flores al igual que la cantidad de polen depositada, pueden afectar negativamente los órganos florales femeninos, reduciendo la producción de semillas. Así mismo, destacan que la polinización natural fue más eficiente, que la artificial, debido a que la flor de esta Solanácea, se enmarca bien en el síndrome de la polinización vibrátil (Buchmann, 1983), ya que sus estambres son de color fuerte; anteras porícidas con granos de polen pequeños y liberados por vibración mecánica directa, que garantiza la constancia de los polinizadores.

Sun et al. (1990), Passam E Khah (1992) y Passam et al. (2001) señalan que las condiciones ambientales, como altas temperaturas y lluvias, que son reinantes en esta región del país y el genotipo, influyen en la formación de frutos, ya que ejercen un efecto separado, pero aditivo, por lo cual, resulta de gran utilidad realizar siembras en las épocas en que el período de floración no coincida con las condiciones ambientales antes anotadas. Las temperaturas extremas pueden influir de manera detrimental sobre el polen y la superficie estigmática u otra estructura del gineceo (Peñaloza, 2001), especialmente, en los primeros cinco días después de la antesis (Sun et al. 1990). 
La mayor cantidad de semillas fue encontrada en flores con frutos provenientes de botones iniciando antesis (corola de color morado), lo que muestra una mejor disposición del estigma el día de la antesis, hecho que concuerda con lo reportado por Kalloo (1993), quien además anotó que hubo mejor respuesta a uno o dos días después de abierta la flor, alcanzando una producción de 1.300 semillas por fruto, en tanto que Rylski et al. (1984) reportan valores entre 572 y 1.639 semillas, resaltando que la longitud de estilo juega un papel importante en la germinación del polen y posterior fecundación de los óvulos. En Capsicum annuum y Serenoa repens, Ofosu-Anim et al. (2006) y Carrington et al. (2003) encontraron receptividad estigmática de dos a cuatro días, señalando que esto varía con la especie y el cultivar (Pessarakli E Dris, 2004). Así mismo, esta variación, por una parte, entre medias de tratamientos puede obedecer a la cantidad y al porcentaje de germinación de granos de polen depositados, lo que afecta la formación de semillas (Rylski et al. 1984; Avila et al. 1989; Polverente et al. 2005; Cardoso, 2005); por otro lado, el efecto de la temperatura sobre el crecimiento del tubo polínico, ya que existen evidencias en tomate (Lohar $\mathcal{E}$ Peat 1998) y en papaya (Pérez - Martínez et al. 2006) que las altas temperaturas conducen a un lento crecimiento del tubo polínico y fallas en el proceso de fecundación, pudiendo causar aborto floral y al efecto del agua (Burke, 2002), puesto que diferencias osmóticas entre el citoplasma del polen y el agua, reducen la cantidad de granos de polen viable por la ruptura de los mismos, lo que ocurre entre pocos segundos y muchos minutos.
En cuanto al índice de semillas, se observaron valores desde 10.732 hasta 214.007 semillas por kilogramo (Tabla 3). Esta variación puede estar relacionada con la deficiencia en los factores de estímulo para la germinación y el crecimiento del tubo polínico, tales como auxinas, giberelinas (Gillaspy et al. 1993) y nutricionales. De la misma manera, es muy posible que algunas flores, por el fenómeno de la heterostília, poseen estilos de longitud variable, factor importante en el éxito de la polinización manual, como lo anotan Nothmann et al. (1983), Kowalska (2006), ya que flores con longitud de estilo medio pueden tener algún grado de fertilidad variable y formar frutos, tanto pequeños como partenocárpicos, de acuerdo a lo acotado por Rylski et al. (1984) y Handique E Sarma (1995).

Las correlaciones entre el peso de fruto y las características del fruto están contenidas en la Tabla 4. Las correlaciones fenotípicas acusaron altos valores, positivos y significativos entre el peso del fruto y número de semillas por fruto e índice de semillas, sugiriendo que el peso de frutos, tiene un efecto directo sobre el número de semillas e índice de semillas, como resultado de la gran cantidad de óvulos fecundados y semillas formadas. De esta manera, en un programa de producción de semillas, el raleo de estructuras reproductivas, a fin de dejar pocos frutos y reducir la competencia entre ellos por los fotosíntatos, para obtener mayor peso es deseable; en caso contrario, se obtendría menor producción de semillas (Tavares et al. 1999; Cavalcanti et al. 2000). El número de semillas por fruto acusó ausencia de correlación con el índice

Tabla 3. Valores medios para las variables peso de fruto (P.F.), número de semillas por fruto (N.S.P.F.) e índice de semillas (I.S.), en hibridaciones artificiales de Solanum melongena $\mathrm{L}$.

\begin{tabular}{|c|c|c|c|}
\hline Tratamiento & $\begin{array}{c}\text { P. F. } \\
\text { (g) }\end{array}$ & $\begin{array}{c}\text { N. S. P. F. } \\
\text { (Número) }\end{array}$ & $\begin{array}{c}\text { I.S. } \\
\text { Semillas } \text { kg }^{-1}\end{array}$ \\
\hline 8 & $235,4 \mathrm{a}^{*}$ & $989,7 \mathrm{a}$ & $214,007 \mathrm{a}$ \\
\hline 7 & $126,8 \mathrm{a}$ & $815,4 \mathrm{a}$ & $130,021 \mathrm{a}$ \\
\hline 5 & $112,1 \mathrm{a}$ & $601,6 \mathrm{a}$ & $112,729 \mathrm{a}$ \\
\hline 6 & $70,7 \mathrm{a}$ & $289,2 \mathrm{a}$ & $110,372 \mathrm{a}$ \\
\hline 4 & $64,5 \mathrm{a}$ & $407,9 \mathrm{a}$ & $127,804 \mathrm{a}$ \\
\hline
\end{tabular}

*Medias con la misma letra no difieren estadísticamente, según la prueba de Bonferroni, $\mathrm{a}=0,05$, estadístico de prueba válido cuando la prueba $\mathrm{F}$ resulta no significativa. 
de semillas, lo que sugiere total independencia entre estas características; además, estas variables no deben ser consideradas como criterio de selección de mejores frutos en un programa de producción de semillas.

Un menor número de semillas por fruto indica mayor tamaño individual, lo que implica un superior contenido de reservas nutricionales y del número de orgánulos subcelulares, que serán asiento para las reacciones bioquímicas que formarán plántulas con gran vigor hasta que éstas sean capaces de subvenir a sus necesidades por medio de la fotosíntesis y la absorción de nutrientes del suelo (Besnier, 1989).

Tabla 4. Coeficientes de correlación estimados entre el peso de fruto y el número de semillas por fruto.

\begin{tabular}{|l|c|c|c|}
\hline & Peso de fruto & Número de semillas por fruto & Índice de semilla \\
\hline Peso de fruto & 1,00 & $0,91^{*}$ & $0,92^{*}$ \\
\hline Número de semillas por fruto & & 1,00 & 0,76 n.s. \\
\hline Índice de semilla & & & 1,00 \\
\hline
\end{tabular}

* Significativo $(P<0,05 \%) ;$ n.s. $=$ no significativo

\section{CONCLUSIONES}

Estados de desarrollo de botones florales con coloración ligeramente lila (próximo a antesis) y lila (iniciando antesis) son óptimos como fuente femenina para realizar hibridación artificial, bien para cruzamiento de padres divergentes o para la producción de semilla híbrida.

El peso y el número de semillas por fruto y el índice de semillas (número de semillas por kilogramo), no fueron afectados por los tratamientos exitosos probados (botones próximos a antesis e iniciando antesis).

El peso del fruto varía de manera conjunta y, en un mismo sentido con el número de semillas por fruto e índice de semillas.

\section{BIBLIOGRAFÍA}

AGRONET. 2008. Producción nacional por producto. Ministerio de Agricultura y Desarrollo Rural. Disponible desde Internet en: http://www.agronet.gov. co (con acceso 26/01/2008).

Acuerdo de competitividad de la cadena de hortalizas, 2006. Ministerio de Agricultura y Desarrollo Rural. Observatorio Agrocadenas Colombia Colombia. Disponible desde Internet en: http://www.Agrocadenas.gov.co (con acceso 30/05/2008).
ANTONINI, A.C.; ROBLES, W.G.; TESSARIOLI, J.N.; KLUGE, R. 2002. Capacidade produtiva de cultivares de berinjela. Hort. Brasileira 20(4):646-648.

ARAMENDIZ, H.; CARDONA, C.; ROBLES, J.; FERNÁNDEZ, C.; HERNÁNDEZ, J. 2006. Polinización cruzada natural en berenjena Solanum melongena L.). Fitotecnia Colombiana. 6(1):59-66.

ARAMENDIZ, H.; HOYOS, F.; GARCIA, E. 1999. Estimación de la variabilidad genética en una población criolla de berenjena (Solanum melongena L.) en el departamento de Córdoba. Temas Agrários. 4(8):117-125.

ÁVILA, C.J.; MARTINHO, M.R.; CAMPOS, J.P. 1989. Polinização e polinizadores na produção de frutos e sementes híbridas de abóbora (Cucurbita pepo var. melopepo). Anais da Soc. Entomol. Brasil. 18(1):13-19.

BESNIER, F. 1989. Semillas: Biología y tecnología. Ediciones Mundi-Prensa. Madrid: España. p.6162, 201-202.

BEZERRA, E.; MACHADO, I. 2003. Floral biology and pollination system of Solanum stramonifolium Jack (Solanaceae) in atlantic forest remnant in Pernambuco. Acta Bot. Brasileira.17(2):247-257. 
BURKE, J.J. 2002. Moisture sensitivity of cotton pollen: an emasculation tool for hybrid production. Agronomy J. 94:883-888.

BUCHMANN, S.L. 1983. Buzz pollination in Angiosperms. In: Jones, C.E.; Little, R.J. (eds). Handbook of experimental pollination biology. Van Nostrand E Reinhold, New York. p.73-113.

BLAT, S.F.; BRAZ, L.T.; ARRUDA, A.S. 2007. Avaliação de híbridos duplos de pimentão. Hort. Brasileira 25(3):350-354.

BLETSOS, F.A. 2002. Evaluation of new and commercial eggplant hybrids and cultivars in relation to their frozen product. Acta Hort. 579:89-93.

BRASIL.1992. Ministério da Agricultura e Reforma Agrária. Regras para análise de sementes. Brasília: SNDA/DNDV/CLAV, 365p.

CARDOSO, A.I.I. 2005. Polinização manual em abobrinha: efeitos nas produções de frutos e de sementes. Hort. Brasileira. 23(3):731-734.

CARDOSO, A.I.I. 2001. Melhoramento de hotaliças. In: NASS, L.L.; CANDEIRA, A.C.; SOARES, I.; VALADARES, M.C. (eds). Recursos genéticos e melhoramento de plantas. Rondonópolis: Fundação MT, cap 12, p.293-326.

CARRINGTON, M.E.; GOTTFRIED, T.D.; MULLAHEY, J.F. 2003. Pollination biology of Saw Palmetto (Serenoa repens) in Southwestern Florida. Int. Palm. Soc. 47:23-27.

CAVALCANTI, R.; CARVALHO DE, L.P.; DOS SANTOS, V.F. 2000. Análise de coeficiente de trilha para os componentes de produção em amendoim. Ciência Agrotecnologia. 24(1):13-16.

DEMIR, I.; ERMIS, S.; OKCU, G.; MATTHEWS, S. 2005. Vigour tests for predicting seedling emergence of aubergine (Solanum melongena L.) seed lots. Seed Science and Technology. 33(2):481-484.

DULBERGER, R.; LEVY, A.; PALEVITCH, D. 1981. Andromonoecy in Solanum marginatum. Botanical Gazette 142:259-262.
FAO, 2006. Organización de las naciones Unidas para la agricultura y la alimentación. Estadísticas sobre agricultura. Disponible desde Internet en: http:// www.faostat.fao.org (con acceso 05/12/2006).

FILGUEIRA, F.A.R. 2000. Novo manual de olericultura: agrotecnologia moderna na produção e comercialização de hortaliças. Viçosa: Universidade Federal de Viçosa, 402p.

GEORGE, R.A.T. 1999. Vegetable seed production. 2.ed. London: CABI Publishing, 219p.

GILLASPY, G.; BEM-DAVID, H.; GRUISSEM, W. 1993. Fruit a development persective. The Plant Cell. 5(10):1439-1451.

GOMES, J.E.; PAVANIZ, M.C.; PERECIN, D.; MARTINS, A.B. 2001. Morfologia floral e biologia reprodutiva de genótipos de aceroleira. Scientia Agrícola. 58(3):519-523.

HANDIQUE A.K.; SARMA A., 1995. Alteration of heterostyly in Solanum melongena L. through gamma-radiation and hormonal treatment. J. Nuc. Agric. Biol. 24:121-126.

JARAMILLO, J.; LOBO, M. 1990. Hortalizas. Manual de Asistencia Técnica $\mathrm{N}^{\circ}$ 28. Ministério de Agricultura y Desarrollo Rural. ICA. División Agropecuária. p.145-163.

KALLOO, G. 1993. Eggplant (Solanum melongena L.). In: Kalloo, G.; Bergh, B.O. (eds.). Genetic improvement of vegetable crops. Pergamon Press Inc, 660 White Plains Road, Tarrytown, New York. p.387-399.

KOHN, J.R.; BARRETT, S.C. 1992. Experimental studies on the functional significance of heterostyly. Evolution. 46:43-55.

KOWALSKA, G. 2006. Eggplant (Solanum melongena L.) flowering and fruiting dynamics depending on pistil type as well as way of pollination and flower harmonization. Folia Horticulturae. 18(1):17-29.

LANTERI, S.; BELLETI, P.; NASSI, M.O., QUAGLIOTTI, L. 1990. Cryo - preservation of pepper and eggplant seeds. Capsicum newsletter 8-9:64-65. 
LEE, E.M.; KIM, K.J.; KIM, H.S.; KIM, W.S. 2002. Improvement of eggplant production through night temperature control related with solar radiation and enhancement of plastic house covering. Acta Hort. 633:Proc.XXVI:287-293.

LOHAR, D.P.; PEAT, W.E. 1998. Floral characteritics of heat-tolerant and heat-sensitive tomato (Lycopersicon esculentum Mill) cultivars at high temperature. Scientia Hort. 73(1):53-60.

MALM, N.R.; RACHIE, K.O. 1971. Setaria millets: A review of the world literature. S.B. 513. University of Nebraska, Lincoln. p.19-29.

MALUF, W.R. 2001. Heterose e emprego de híbridos F1 em hortaliças. In: Nass, L.L.; Candeira, A.C.; Soares, I.; Valadares, M.C. (eds). Recursos genéticos e melhoramento de plantas. Rondonópolis: Fundação MT, 2001. cap 13, p.327-355.

MOTTEN, A.; STONE, J. 2000. Heritability of stigma position and the effect of stigma-anther separation on outcrossing in a predominantly self-fertilizing weed, Datura stramonium (Solanaceae). Am. J. Bot. 87(3):339-347.

MUNIZ, F.B.; DA SILVA, M.A.; MELLO, F.A.; MAIA, M.S. 2001. Avaliação da qualidade de sementes de melão cv. Gaúcho produzidas pelo sistema agroecológico. Horticultura Brasileira, Brasília, 19, suplemento. CD-ROM, julho 2001.

NOTHMANN, J.; RYLSKI, L.; SPIGELMAN, M. 1983. Interactions between floral morphology position in cluster and 2, 4-D treatment in three eggplant cultivars. Scientia Hort.22:35-44.

OFOSU-ANIM, J.; OFFEL, S.K.; YAMAKI, S. 2006. Pistil receptivity, pollen tube growth and gene expression during early fruit development in sweet pepper (Capsicum annuum). Internal. J. Agriculture $\mathcal{E}$ Biology. 8(5):576-579.

PALENCIA, G.; MERCADO, T.; COMBATT, E. 2006. Estudio Agrometeorològico del Departamento de Córdoba. Facultad de Ciencias Agrícolas, Universidad de Córdoba. Gráficas del Caribe. 126p.
PASSAM, H.C.; BALTAS, C.; BOYIATZOGLOU, A.; $\mathrm{KHAH}$, E.M. 2001. Flower morphology and number of aubergine (Solanum melongena L.) in relation to fruit load and auxin application. Sci. Hort. 89(4):309-316.

PASSAM, H.C.; KHAH, E.M. 1992. Flowering, fruit set and fruit and seed development in two cultivars of aubergine (Solanum melongena L.) grown under plastic cover. Sci. Hort. 51(3-4):179-185.

PEÑALOZA, P. 2001. Semillas de hortalizas: manual de producción. Ediciones Universitarias de Valparaíso de la Universidad Católica de Valparaíso. Editorial Salesianos Impresores S.A. 147p.

PÉREZ, D. 2006. Estudio sobre la biología floral de la berenjena (Solanum melongena L). Trabajo de grado Ingeniería Agronómica. Facultad de Ciencias Agrícolas, Universidad de Córdoba. 72p.

PEREZ-MARTÍNEZ, J.; BASSO, C.; JAUREGUI, D.; MELENDEZ, L. 2006. Cantidad, viabilidad y germinabilidad de los granos de polen de Carica papaya L. Revista de la Facultad de Agronomía - LUZ. 23(2):172-180.

PESSARAKLI, M.M.; DRIS, R. 2004. Pollination and breeding of eggplants. Food, Agr. E Environ. 2(1):218-219.

POLVERENTE, M.R.; CARNEIRO, D.; CARDOSO, A.I. 2005. Produção e qualidade de sementes de berinjela em função do horário de polinização manual. Bragantia. 64(3):467-472.

PROHENS, J.; BLANCA, J.M.; NUEZ, F. 2005. Morphological and molecular variation in a collection of eggplants from a secondary center od diversity: Implications for conservation and breeding. J. Am. Soc. Hort. Sci. 130(1):54-63.

RYLSKI, I.; NOTHMANN, J.; ARCAN, L. 984. Differential fertility in short-styled eggplant flowers. Scientia Hort. 22(1):39-46.

SEKARA, A.; CEBULA, S.; KUNICKI, E. 2007. Cultivated eggplants - origin, breeding, objectives and genetic resources, a review. Folia Hort.19(1):97-114. 
SILES, M.M.; BALTENSPERGER, D.D.; NELSON. L.A. 2001. Technique for Artificial Hybridization of Foxtail Millet [Setaria italica (L.) Beauv.]. Crop Science. 41(5):1408-1412.

SOUSA, J.A.; MALUF, W.R.; GOMES, L.A.A. 1997. Produtividade e qualidade de frutos de cultivares de polinização aberta e híbrida $\mathrm{F}_{1}$ de berinjela (Solanum melongena L.). Ciência e Agrotecnologia. 21(2):334-342.

SUN, W.; WANG, D.; WU, Z.; ZHI, J. 1990. Seasonal change of fruit setting eggplants (Solanum melongena L.) caused by different climatic conditions. Scientia Hort. 51(1-2):55-59.

SWARUP, V. 1995. Genetic resources and breeding of aubergine (Solanum melongena L.). Acta Hort. 412:71-79.
TAVARES, M.; TAVARES DE MELO, A.M. ; SCIVITTARO, W.B.1 99. Efeitos diretos e indiretos e correlações canônicas para caracteres relacionados com a produção de pimentão. Bragantia. 58(1):41-47.

TEXEIRA, L.C.; MACHADO, I.C. 2004. Biologia da polinização e sistema reprodutivo de Psychotria barbiflora DC. (Rubiaceae). Acta Bot. Bras. 18(4):853-862.

VALLEJO, F.; ESTRADA, E. 2002. Mejoramiento genético de plantas. Universidad Nacional de Colombia, Sede Palmira. Feriva S.A. 402p.

Recibido: Febrero 26 de 2008

Aceptado: Julio 21 de 2008 